Jacqueline M. Leung MD, * Darwin A. Pastor†

\title{
Dissociation between haemodynamics and sym- pathetic activation during anaesthetic induction with desfluranes
}

Purpose: To compare the simultaneous haemodynamic effects, sympathetic activation and cardiac risks associated with desflurane used in a balanced technique, with those of isoflurane anaesthesia.

Methods: A prospective, randomized, open label study was conducted at a University medical centre. Forty patients undergoing major non-cardiac surgery were randomized to receive either desflurane or isoflurane as the primary anaesthetic agent. After premedication, fentanyl and thiopentone were administered iv. Anaesthesia was increased up to $1.0 \mathrm{MAC}_{\mathrm{E}}$ in $\mathrm{O}_{2}$ via controlled mask ventilation and maintained at I.O MAC before tracheal intubation. Maintenance consisted of $\mathrm{N}_{2} \mathrm{O}_{1} \mathrm{O}_{2}$ and desflurane or isoflurane for $10 \mathrm{~min}$. During the study, HR and arterial BP were continuously measured, as were ECG ST-segments and ventricular dysrhythmias using a 3-channel Holter ECG recorder. Left ventricular global and regional function were measured using precordial echocardiography. Serial plasma catecholamine concentrations were measured.

Results: For both groups, HR was maintained without increases over baseline values while systolic BP showed a progressive decrease during induction. Use of beta blockade during induction was higher in the desflurane $(7 / 20=35 \%)$ than in the isoflurane group $(\mid / 20=5 \%), P=0.04$. The plasma norepinephrine concentrations progressively increased in the desflurane group but not in the isoflurane group. Four patients in the desflurane and three in the isoflurane group developed transient worsening of regional function but no change in mean left ventricular ejection fraction area and no ECG ischaemia occurred during anaesthetic induction.

Conclusions: Desflurane differs from isoflurane in that sympathetic stimulation persisted despite blunting of potential hyperdynamic haemodynamic responses by narcotic and beta blockade. However, this sympathetic activation did not appear to increase cardiac risks.

Objectif : Comparer simultanément les effets hémodynamiques, l'activation sympathique et les risques cardiaques associés à l'utilisation du desflurane ou de l'isoflurane utilisés selon une technique anesthésique balancée.

Méthodes : Une étude prospective, randomisée et ouverte a été menée dans un centre hospitalo-universitaire. Quarante patients devant subir une chirungie majeure non cardiaque ont été répartis de façon aléatoire en deux groupes, l'un recevant du desflurane et l'autre de l'isoflurane comme agent anesthésique principal. Après une prémédication, on a procédé à l'injection intraveineuse de fentanyl et de thiopental. L'anesthésie a été augmentée jusqu'à I,0 MAC $\mathrm{Evec}_{2} \mathrm{O}_{2}$ à l'aide d'une ventilation contrôlée par masque et maintenue par la suite à I,O MAC avant l'intubation trachéale. Le maintien était assuré par l'utilisation du $\mathrm{N}_{2} \mathrm{O}$, de l'oxygene et de l'isoflurane ou du desflurane pendant 10 minutes. Durant l'étude, la FC, et la TA ont été mesurées en continu, de même le segment ST à l'ECG et les dysrythmies ventriculaires qui ont été enregistrées à l'aide d'un moniteur ECG de Holter à 3 canaux. Les fonctions ventriculaires gauches globales et régionales ont été évaluées en utilisant un échocardiographe précordial. Des concentrations plasmatiques sériées de catécholamines ont été mesurées.

Résultats : Pour les deux groupes, la FC s'est maintenue sans augmentation par rapport aux valeurs de départ, alors que la TA systolique décroissait progressivement durant la phase d'induction. L'utilisation de bloqueurs durant l'induction était plus fréquente dans le groupe sous desflurane $(7 / 20=35 \%)$ que dans le groupe sous isollurane $(1 / 20=5 \%), P=0,04$. Les concentrations plasmatiques de norépinéphrine ont augmenté progressivement dans le groupe desflurane mais non dans le groupe isoflurane. Quatre patients sous desflurane et trois sous isoflurane ont développé une dégradation transitoire de la fonction régionale, mais aucun changement ne s'est produit au niveau de la fraction moyenne d'éjection du ventricule gauche et aucune ischémie à l'ECG n'est survenue pendant l'induction de l'anesthésie.

Conclusion : Le desflurane diffère de l'isoflurane en ce que la stimulation sympathique persiste malgré la tentative d'émousser les réponses hémodynamiques hyperdynamiques potentielles par l'usage d'un narcotique et d'un bêta bloqueur. Cependant, cette activation sympathique n'a pas semblé accrôtre les risques de problèmes cardiaques.

From the Department of Anaesthesia, University of California, San Francisco. Supported in part by the Pretenure award and a research award from the Academic Senate, University of California, San Francisco.

Address correspondence to: Dr. J.M. Leung, University of California, San Francisco. Mount Zion Medical Center, Department of Anaesthesia, 1600 Divisadero Street, San Francisco, CA 94.115. E-mail: jmleung@itsa.ucsf.edu

Accepted for publication February 11, 1998. 
I NDUCTION of anaesthesia with desflurane (without adjuvants except for thiopentone) is associated with greater haemodynamic change and a higher incidence of myocardial ischaemia in patients undergoing coronary artery bypass graft surgery than is induction with sufentanil.1 In healthy volunteers, "titration of desflurane following thiopentone induction and increasing the concentration of desflurane from 1.0 to 1.5 MAC result in sympathetic excitation (measured by peroneal nerve discharge), hypertension and tachycardia". 2 This transient sympathetic stimulation can be partially modulated in volunteers by concurrent administration of small doses of opioid and/or beta-blocking drugs, or the alpha 2 agonist, clonidine. 3 In small groups of patients undergoing cardiac surgery, desflurane given in conjunction with larger doses of opioids (10 $\mu \mathrm{g} \cdot \mathrm{kg}-1$ fentanyl) showed similar haemodynamic profile as isoflurane/narcotic or high dose narcotic techniques.4,5 However, whether the haemodynamic changes and/or sympathetic activation can be ablated resulting in lower cardiac risks in patients undergoing major surgery has not been determined.

Therefore, the objectives of our study were: 1) to determine if haemodynamic perturbations and sympathetic activation associated with desflurane use could be ablated, and 2) to determine if the associated cardiac risks as measured by ECG ST-segment changes, left ventricular dysfunction and ventricular dysrhythmias were lowered. We employed a standardized anaesthetic which consisted of the use of an opioid and avoidance of rapid increases in the inspired concentration of desflurane (isoflurane as control). We hypothesized that ablation of haemodynamic aberrations should result in similar cardiac risks in patients undergoing anaesthetic induction with desflurane compared to those receiving isoflurane.

\section{Materials and methods}

After approval by the institutional human research committee and informed consent obtained from each patient, 40 adult patients undergoing major non-cardiac surgery requiring general anaesthesia and an arterial catheter for clinical management were studied between April, 1994 and May 1996 at the University of California, San Francisco.Mount Zion Medical Center. The decision to employ invasive arterial blood pressure monitoring was up to the discretion of the treating anesthesiologists and was not controlled by study protocol. The exclusion criteria were patients with increased intra-cranial pressure, unstable angina, congestive heart failure, pregnancy, preoperative haematocrit $<30 \%$, or conditions (e.g., pacemaker, left bundle branch block) precluding the use of electrocardiography (ECG) for detection of ischaemia. The study was an open-label, prospective, randomized design in which patients were randomized to receive either desflurane or isoflurane (control) as the primary anaesthetic agent. Patients were premedicated with up to $0.07 \mathrm{mg} \cdot \mathrm{kg}^{-1}$ iv midazolam, approximately $30 \mathrm{~min}$ before anaesthetic induction. After preoxygenation, they received up to $1.5 \mathrm{\mu g} \cdot \mathrm{kg}^{-1}$ fentanyl, and up to $7 \mathrm{mg} \cdot \mathrm{kg}^{-1}$ thiopentone, to achieve loss of consciousness. Vecuronium, 0.1 $\mathrm{mg} \cdot \mathrm{kg}^{-1} i v$, was used for muscle relaxation. The depth of anaesthesia was increased gradually to $1.0 \mathrm{MAC}$ endtidal concentration of vapor (1.15\% for isoflurane and $6 \%$ for desflurane) in oxygen via controlled ventilation by mask, and was maintained at 1.0 MAC before tracheal intubation. The inhalational agents were increased step-wise to permit the inspired concentration of desflurane to be increased by $1 \%$ and isoflurane by $0.25 \%$ every 4-5 breaths. Ventilation was controlled to maintain $\mathrm{PaCO}_{2}$ between $35-45 \mathrm{mmHg}$. Anaesthesia was maintained with nitrous oxide $60 \%$, oxygen $40 \%$ and desflurane or isoflurane for $10 \mathrm{~min}$ after tracheal intubation. Routine monitoring included invasive measurement of systemic blood pressure, heart rate and rhythm by 5 -lead ECG and oxygen saturation by pulse oximetry. End-tidal carbon dioxide and inspiratory and expiratory desflurane or isoflurane concentrations throughout the study were measured with the Datex capnography monitor (Datex Ultima, Tewksbury, MA). Specialized monitoring included Holter ECG and precordial echocardiography.

The anesthesiologists were requested to maintain heart rate not exceeding $20 \%$ above, and systolic blood pressure within $\pm 20 \%$ of preoperative baseline values throughout the study period (from the administration of fentanyl to $10 \mathrm{~min}$ after tracheal intubation) using vasoactive agents (neosynephrine or ephedrine) or beta-adrenergic blocking agents, as needed. The administration of vasopressors and beta adrenergic blocking agents was recorded.

\section{Haemodynamics}

Intraoperatively, systolic, mean and diastolic blood pressure were continuously monitored using radial artery catheters in all patients. Heart rates were derived from the clinical ECG recordings. Systolic, diastolic, mean arterial pressure, and heart rate were measured continuously (averaged every $60 \mathrm{~s}$ ) on entry into the operating room and stored in a lap-top computer (Toshiba T1200 XE, Toshiba Corporation, Tokyo, Japan). The zero reference point of the blood pressure transducer was located $5 \mathrm{~cm}$ posterior to the sternal angle in a direction perpendicular to the frontal 
plane of the chest. The time clocks on the clinical haemodynamic monitor, Holter monitor and echocardiographic system were synchronized before anaesthetic induction.

\section{Electrocardiography}

All patients were continuously monitored using a three-channel Holter ECG recorder (Del Mar model 459, Del Mar Avionics, Irvine, CA). ECG monitoring began preoperatively, before induction of anaesthesia and continued throughout surgery. The frequency response of the Holter recorder met the American Heart Association specification for ST changes, the cutoff limit being $0.05 \mathrm{~Hz}$ for low frequency and 100 $\mathrm{Hz}$ for high frequency. For Holter monitoring, three bipolar leads, CC5, modified CM5 and ML, were used. Each ECG recording on Holter tapes was scanned visually using an ECG analysis system (Del Mar Model 750). All normal QRS complexes were identified, and all abnormal QRS complexes (e.g., ventricular ectopic beats and conduction abnormalities) were excluded. Continuous ST-segment trends were generated for the entire tape.

All possible ischaemic episodes were reviewed and verified by investigators who were blinded to patient identity and outcome. An ischaemic episode was defined as a reversible ST-segment shift from baseline of $0.1 \mathrm{mV}$ depression at $J+60 \mathrm{msec}$, or $0.2 \mathrm{mV}$ elevation at the $J$ point lasting for at least one minute. The time after the J point chosen to measure ST-segment depression was adjusted to exclude $T$ wave during tachycardia.

After ST analysis, all Holter tapes were re-scanned for the occurrence of episodes of ventricular tachycardia ( 3 beats) and premature ventricular contractions. Each ventricular ectopic beat identified by the Holter analysis machine was visually reviewed on the scanner and subsequent continuous hard-copies of the ECG strips printed for validation by a second reviewer. The number of premature ventricular contractions or ventricular tachycardic episodes per minute monitored was compared with each patient's individual preoperative baseline. In addition, between group (desflurane and isoflurane) comparisons was also made.

\section{Echocardiography}

Precordial echocardiography was performed during the induction period to assess global and regional left ventricular function. Immediately following the application of the face mask for preoxygenation, a 2.5MHZ phased array transducer (Hewlett-Packard) was positioned on the left hemithorax (patient in the supine position) and maintained at the level of the mid-papillary muscles to obtain a short-axis view of the left ventricle. Precordial data were recorded and analyzed off-line for measurement of left ventricular ejection fraction area and regional wall-motion and wall-thickening abnormalities. Ejection fraction area (EFA) was calculated as: $\mathrm{EFA}=[(\mathrm{EDA}-\mathrm{ESA}) / \mathrm{EDA}]$ $\times 100 \%$ where EDA represents the end-diastolic area and ESA, the end-systolic area. ${ }^{6,7}$ Regional wallmotion at the level of the short axis was measured by blinded investigators using a scoring system of 1-5 indicating hyperkinesis, normal wall-motion, hypokinesis, akinesis or dyskinesis. Significant regional dysfunction was defined as any regional wall-motion or thickening deterioration of 1 grade from normal wallmotion. The segments of the left ventricle were divided according to recommendations by the American Society of Echocardiography. ${ }^{8}$ Inter and intra-observer variability in the interpretation of regional wallmotion abnormalities has previously been reported. ${ }^{9}$

\section{Plasma catecholamine measurement}

Blood was sampled from the radial arterial catheter for measurement of plasma catecholamine concentrations before anaesthetic induction (conscious baseline), at 1.0 MAC end-tidal concentration of vapor, and at 1,5 and 10 min after tracheal intubation. Plasma was separated in a refrigerated centrifuge at $4 \mathrm{C}$ within $30 \mathrm{~min}$ after blood was drawn, then frozen immediately at $-70 \mathrm{C}$. The specimens were shipped to an outside laboratory (Specialty Laboratories, Santa Monica, CA) for measurement of epinephrine, norepinephrine and dopamine concentrations using high-pressure liquid chromatography. The limits of detection for epinephrine were $10-2,000 \mathrm{pg} \cdot \mathrm{mL}^{-1}$, norepinephrine $5-2,000$ $\mathrm{pg} \cdot \mathrm{mL}^{-1}$ and dopamine $1-1,000 \mathrm{pg} \cdot \mathrm{mL}^{-1}$. Coefficients of variation for intra-assay precision were $6.0-6.5 \%$ for epinephrine, $3.5-5.5 \%$ for norepinephrine and $7.0-8.0 \%$ for dopamine. Those for inter-assay precision were 3.18.3\% for epinephrine, $2.34-6.21 \%$ for norepinephrine and $3.45-9.19 \%$ for dopamine. Sample values less than the limit of detection were considered to indicate a concentration just below the limit of detection.

\section{Statistical analysis}

Data from each anaesthetic group compared with baseline conscious values were analyzed using paired $t$-tests with Bonferroni correction. Chi-square analysis or Fisher's Exact Test with continuity correction was applied to categorical data. Student's $t$ test was used to test the difference between the means in the two groups. Differences in heart rates and blood pressure within group were assessed by two-way analysis of variance using repeated measures. All data are reported as mean \pm SD. Statistical significance was accepted at $P<$ 0.05 . 


\section{Results}

The demographics of the patients are shown in Table I. Most were elderly, with the majority undergoing vascular or urological surgery. Ten percent of the patients had documented coronary artery disease and $52.5 \%$ of the patients had risk factors for coronary artery disease. There were no differences between groups, and no study-related complications occurred in any patient. The doses of adjuvant agents given during anaesthetic induction were not different between the two anaesthetic groups except for slightly more fentanyl given to the desflurane group (Table II). The mean duration of induction (up to reaching $1 \mathrm{MAC}$ end-tidal concentration of the anesthetic) between the two inhalational agents also was not different: $5.7 \pm 1.3 \mathrm{~min}$ in the desflurane persus $7.1 \pm 3.1 \mathrm{~min}$ in the isoflurane group, $P$ $=0.12$.

\section{Haemodynamics}

For both anaesthetic groups, heart rates were maintained without increases over preoperative baseline values, except for lower heart rates at $10 \mathrm{~min}$ after tracheal intubation in both groups, compared with conscious baselines (Figure 1). The control of heart rate (to maintain HR to $<20 \%$ elevation from preoperative baseline values) was achieved with a greater use of beta adrenergic blockade during induction with desflurane $(7 / 20=35 \%)$ than with isoflurane $(1 / 20=5 \%), P=$ 0.04 . During induction, systolic blood pressure was unchanged in both groups before tracheal intubation, then decreased progressively in both groups following

TABLE 1 Demographic data

\begin{tabular}{lll}
\hline & $\begin{array}{l}\text { Isoflurane } \\
(n=20)\end{array}$ & $\begin{array}{l}\text { Desflurane } \\
(n=20)\end{array}$ \\
\hline Age in years & $67 \pm 12$ & $64 \pm 12$ \\
Sex (male/female) & $50 \% / 50 \%$ & $45 \% / 55 \%$ \\
History of coronary artery disease & $4(20 \%)$ & $0(0 \%)$ \\
Hypertension & $12(60 \%)$ & $8(40 \%)$ \\
Diabetes mellitus & $4(20 \%)$ & $4(20 \%)$ \\
Smoking & $1(5 \%)$ & $3(15 \%)$ \\
Hypercholesterolaemia & $3(15 \%)$ & $2(10 \%)$ \\
& & \\
Preoperative cardiac medications & & \\
Calcium channel blocking agents & $4(20 \%)$ & $5(25 \%)$ \\
Beta adrenergic blocking agents & $2(10 \%)$ & $1(5 \%)$ \\
Anti-hypertensive agents & $3(15 \%)$ & $1(3 \%)$ \\
& & \\
Type of surgery & & \\
General & $3(15 \%)$ & $3(15 \%)$ \\
Urologic & $6(30 \%)$ & $6(30 \%)$ \\
Vascular & $9(45 \%)$ & $7(35 \%)$ \\
Neurosurgical & 0 & $1(5 \%)$ \\
Orthopedic & $2(10 \%)$ & $2(10 \%)$ \\
\hline
\end{tabular}

tracheal intubation (Figure 2). The use of vasopressors (ephedrine or neosynephrine) did not differ between groups.

\section{Catecholamine concentrations}

Plasma norepinephrine concentrations progressively increased during induction and after tracheal intubation in the desflurane group but remained stable in the isoflurane group (Figure 3). Epinephrine concentrations increased at one minute after intubation in the desflurane group but were unchanged in the isoflurane group (Figure 4). There was no change in dopamine concentration in either group during anaesthetic induction (Figure 5).

\section{Ventricular function, ECG changes and ventricular dysrhythmias}

Thirty three of 40 patients ( $82.5 \%$ ) studied had usable echocardiographic images for analysis of global and regional left ventricular function. Seven patients $(4 / 15=27 \%$ in the desflurane group and $3 / 18=$ $16.7 \%$ in the isoflurane group, $P=0.40$ ) developed transient worsening of regional wall-motion during anaesthetic induction. No patient developed ECG changes suggestive of ischaemia during anaesthetic induction. The number of ventricular ectopic beats occurring during induction was similar between the two anaesthetic groups (Table III). Overall, no change in mean left ventricular ejection fraction area occurred in either group throughout anaesthetic induction period (Figure 6). However, two patients in each group did develop transient worsening of left ventricular ejection fraction area of $>10 \%$ over the awake baseline values during induction.

\section{Discussion}

Our results are in accordance with those reported in studies of healthy volunteers demonstrating that desflurane differs from isoflurane in that sympathetic activation, as manifest by elevation in norepinephrine concentrations, occurs during induction of anaesthesia despite the blunting of potential hyperdynamic haemodynamic responses by concurrent administration of a small dose of narcotic and beta blockade. However, despite this persistent sympathetic activation, cardiac risks as measured by global and regional left ventricular function, ECG ST-segment changes and ventricular dysrhythmias, did not appear to increase in the desflurane group.

One of the early studies in human volunteers revealed that sympathetic stimulation may occur with desflurane. ${ }^{10}$ These investigators suggested that the increases in heart rate, blood pressure and cardiac out- 
TABLE II Anaesthetic induction drug data

\begin{tabular}{llll}
\hline & Isoflurane $(\mathrm{n}=20)$ & Desfurane $(\mathrm{n}=20)$ & $P$ \\
Sodium thiopental $(\mathrm{mg})$ & $253 \pm 104$ & $\mathbf{2 2 6} \pm 80$ & 0.46 \\
Midazolam $(\mu \mathrm{g})$ & $1.61 \pm 1.12$ & $1.83 \pm 0.91$ & 0.40 \\
Fentanyl $(\mu \mathrm{g})$ & $103 \pm 24$ & $123 \pm 33$ & 0.04 \\
Vecuronium $(\mathrm{mg})$ & $7.5 \pm 2.4$ & $7.7 \pm 2.7$ & 0.96 \\
& & & \\
Vasopressors & $(\mathrm{n})$ & $(\mathrm{n})$ & 0.16 \\
Beta adrenergic blocking agents & $3 / 20(15 \%)$ & $8 / 20(40 \%)$ & 0.04 \\
\hline
\end{tabular}

TABLE III Incidence of ventricular dysrhythmias

\begin{tabular}{llll}
\hline & Isoflurane & Desflurane & $P$ value \\
\hline $\begin{array}{l}\text { Preinduction (baseline) } \\
\text { \# patients with PVC }\end{array}$ & 5 & 5 & $>0.99$ \\
\#PVC/min monitoring & $0.30 / \mathrm{min} \pm 0.62 / \mathrm{min}$ & $1.59 / \mathrm{min} \pm 2.59 / \mathrm{min}$ & 0.31 \\
& & & 0.24 \\
Induction & 2 & 6 & 0.57 \\
\# patients with PVC & $0.27 / \mathrm{min} \pm 0.19 / \mathrm{min}$ & $0.59 / \mathrm{min} \pm 0.72 / \mathrm{min}$ & \\
\#PVC/min monitoring & & & \\
\hline
\end{tabular}

PVC $=$ premature ventricular ectopic beats. In order to adjust for the slightly different monitioring times among patients, the number of PVC's observed was divided by the duration of monitoring in minutes. No patient had ventricular tachycardia ( 23 beats).

put during desflurane anaesthesia with oxygen "are similar to those observed after administration of norepinephrine or epinephrine or direct stimulation of sympathetic outflow". The clinical implications of such changes were not apparent until the study conducted by Helman et al. ${ }^{1}$ who demonstrated that anaesthetic induction with desflurane (without adjuvants except for premedications, thiopentone and vecuronium) was associated with more haemodynamic changes (tachycardia, systemic and pulmonary hypertension) and myocardial ischaemia than was induction with sufentanil in patients undergoing coronary artery bypass graft surgery. These results were confirmed by a subsequent study in human volunteers demonstrating that titration of desflurane following thiopentone induction and increasing the concentration of desflurane from 1.0 to 1.5 MAC result in sympathetic excitation (measured by peroneal nerve discharge), hypertension and tachycardia. ${ }^{2}$

Critics of the study by Helman et al. have often suggested that desflurane in oxygen without narcotic adjuvant was an "unusual anaesthetic technique". 5 Since the main goal of the study by Helman et al. was to determine the pure effects of desflurane on systemic and coronary circulation, delivery of a substantial amount of narcotic would have confounded their results. It is not surprising that desflurane, when given with a background dose of $10 \mu \mathrm{g} \cdot \mathrm{kg}^{-1}$ fentanyl, ${ }^{4}$ produced stable haemodynamics comparable to that achieved with isoflurane anaesthesia in 41 patients undergoing coronary artery bypass graft surgery.

The controversy surrounding the safety of desflurane and its ability to cause sympathetic stimulation and potential associated increases in myocardial ischaemia prompted many subsequent studies in human volunteers in an attempt to determine the etiologic and mitigating factors. Using split-lung ventila-

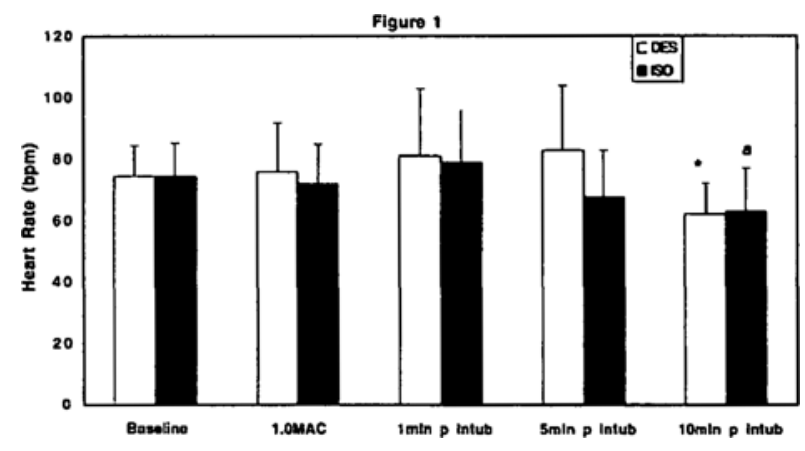

FIGURE 1 Heart rates (mean \pm SD) measured at preoperative baseline, at end-tidal concentration of $1 \mathrm{MAC}$ of the vapor, at 1,5 and $10 \mathrm{~min}$ after tracheal intubation for the two anaesthetic groups are shown. For all figures, $\mathrm{p}$ intub $=$ post intubation, $\mathrm{DES}=$ desflurane, ISO $=$ isoflurane.

* indicates significantly different from preoperative baseline values in the desflurane group,

a indicates significantly different from preoperative baseline values in the isoflurane group. 


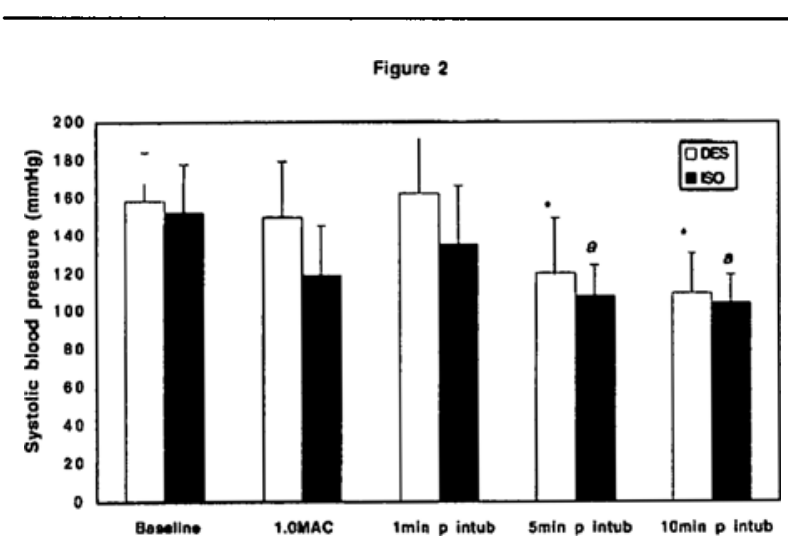

FIGURE 2 Systolic blood pressure (mean \pm SD) measured at preoperative baseline, at end-tidal concentration of $1 \mathrm{MAC}$ of the vapor, at 1,5 and $10 \mathrm{~min}$ after tracheal intubation for the two anaesthetic groups are shown.

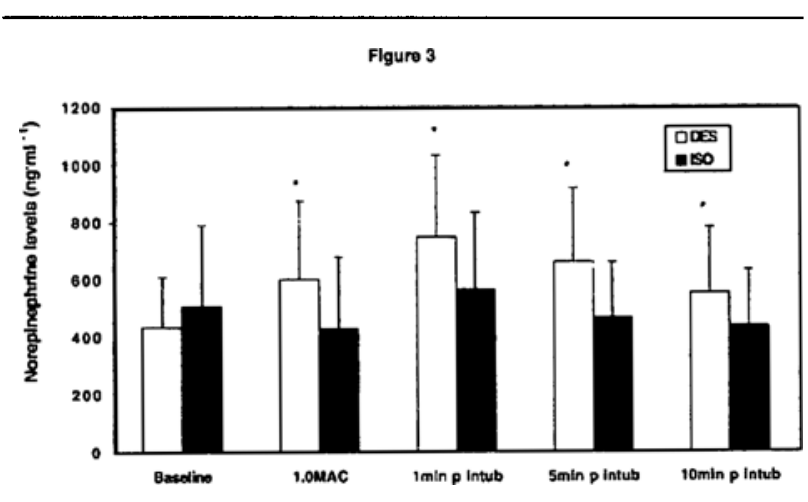

FIGURE 3 Plasma norepinephrine levels (mean \pm SD) measured at preoperative baseline, at end-tidal concentration of 1 MAC of the vapor, at 1,5 and $10 \mathrm{~min}$ after tracheal intubation for the two anaesthetic groups are shown.

tion, selective airway stimulation by desflurane to either the right lung or the left lung, with unchanged systemic desflurane concentrations, increases both heart rate and blood pressure. ${ }^{11,12}$ These results suggest that sites within the lungs, separate from systemic sites (and the upper airway), mediate sympathetic stimulation by desflurane. ${ }^{12}$ Further evidence that systemic sites also may be involved in sympathetic stimulation is demonstrated by numerous reports that neither topical, nebulized or intravenous lidocaine (or lignocaine) abolish the sympathetic or haemodynamic changes associated with rapid increases in desflurane concentrations. ${ }^{12-14}$

Our current results agree with those in human volunteers demonstrating that desflurane-induced tran- sient increases in blood pressure and heart rate during induction of anaesthesia can be modulated by concurrent administration of small doses of opioids and beta blockade. ${ }^{15}$ However, we also found that, as reported in volunteers, ${ }^{2,3}$ despite a similar reduction in blood pressure, circulating norepinephrine concentrations are greater in patients receiving desflurane than in those given isoflurane. Whether larger doses of opioids can obliterate the sympathetic excitation associated with desflurane has not been conclusively determined. Even if proven efficacious, a technique combining desflurane with large doses of opioid will be limited to surgical cases of relatively longer duration because of the respiratory depressant effect of the narcotic, excluding the usefulness of this technique to ambulatory surgical patients. Other agents, such as esmolol, clonidine and propofol, only partially block this sympathetic activation or have undesirable side effects. ${ }^{3,16}$

The clinical importance of sympatheric excitation without blood pressure and heart rate elevation is unclear. In our study, no patient developed ECG changes suggestive of ischaemia during the study period and the incidence of global left ventricular dysfunction was similarly low between groups. However, the incidence of regional ventricular dysfunction and ventricular dysrhythmias tended to be higher in the desflurane group. Therefore, it is possible that a small difference in cardiac risks may exist between desflurane and isoflurane induction in "higher risk" patients such as those with definite coronary artery disease since only $10 \%$ of our study subjects had definite coronary artery disease, although 53\% had risk factors. Moreover, a the-

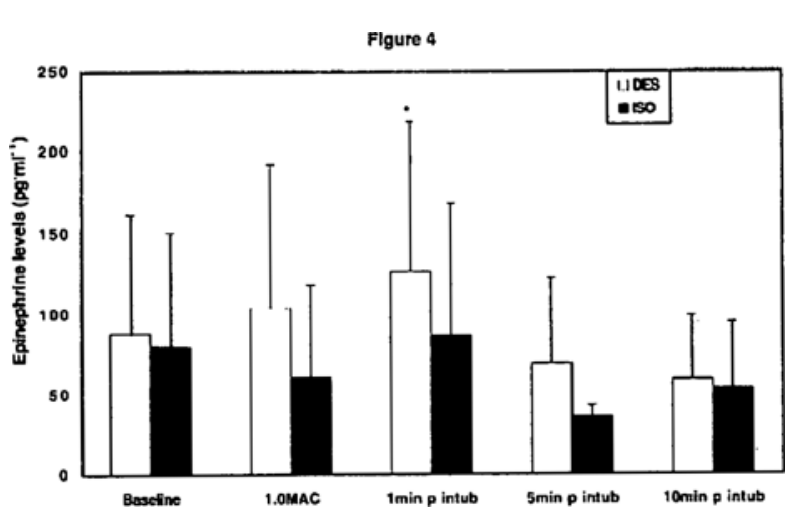

FIGURE 4 Plasma epinephrine levels (mean $\pm \mathrm{SD}$ ) measured at preoperative baseline, at end-tidal concentration of $1 \mathrm{MAC}$ of the vapor, at 1,5 and $10 \mathrm{~min}$ after tracheal intubation for the two anaesthetic groups are shown. 


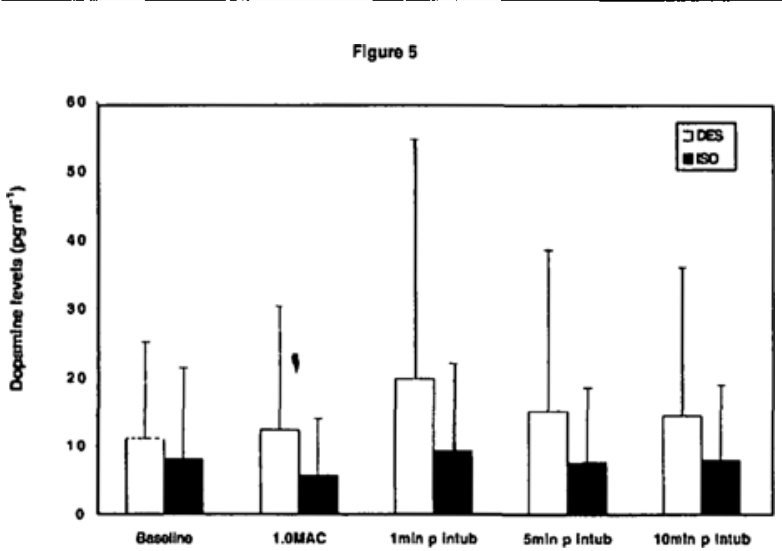

FIGURE 5 Plasma dopamine levels (mean \pm SD) measured at preoperative baseline, at end-tidal concentration of $1 \mathrm{MAC}$ of the vapor, at 1,5 and 10 min after tracheal intubation for the two anaesthetic groups are shown.

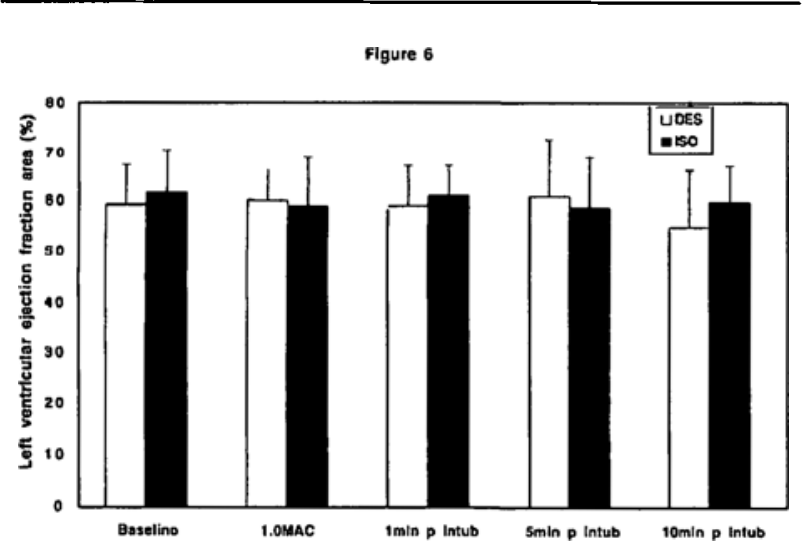

FIGURE 6 Left ventricular ejection fraction area (mean \pm SD) measured at preoperative baseline, at end-tidal concentration of 1 MAC of the vapor, at 1,5 and $10 \mathrm{~min}$ after tracheal intubation for the two anaesthetic groups are shown.

oretical concern exists that sympathetic stimulation may directly increase myocardial oxygen demand and adversely affect the coronary microcirculation. ${ }^{17}$ However, the clinical importance of such potential adverse but relatively transient changes is not immediately apparent. Extrapolating our present results and those from previous studies to clinical practice, the sympathetic activation associated with desflurane induction may be more completely abolished with pretreatment of larger doses of opioid, -2 agonists, intravenous induction agents or -adrenergic blocking agents. However, the costs and labour intensity associated with such a technique limits the usefulness of desflurane as an induction agent compared to other inhalational agents.

Several potential limitations exist in our study. First, the use of vasoactive agents to maintain blood pressure and heart rate might have affected plasma catecholamine measurements. However, catecholamine levels did not differ between those who received and those who did not receive vasopressors during anesthetic induction. Second, whether the "dissociation" between haemodynamics and catecholamine elevation can be obliterated by higher doses of beta antagonists cannot be determined from this study. Third, our study was not designed to address whether cardiac end-points such as myocardial infarction are different between desflurane and isoflurane anaesthesia. Although there is some clinical evidence suggesting that catecholamine levels during anaesthetic emergence from peripheral vascular surgery may be related to adverse cardiac outcomes and vascular graft occlusion, ${ }^{18}$ there is no evidence from our data to speculate that transient elevation of catecholamine levels during anaesthetic induction with desflurane may increase cardiac risk such as myocardial infarction.

In summary; our results demonstrate that haemodynamic activation during gradual increase in desflurane concentrations can be prevented with opioid pretreatment and further suppression with beta blockade. However, a dissociation exists between haemodynamics and catecholamine concentrations, i.e., despite blunting of hyperdynamic haemodynamic responses, plasma catecholamine concentrations increase. This persistent sympathetic activation did not appear to increase cardiac risks.

\section{Acknowledgment}

The authors thank the anesthesiologists (faculty and residents) at UCSF.Mount Zion Medical Center, in particular, Joseph Romson PhD MD for their assistance. We are appreciative of the helpful editorial service of Winifred von Ehrenburg and the excellent technical assistance of Carlos Gonzalez MD, and Ricardo Charles RN.

\section{References}

1 Helman JD, Leung JM, Bellows WH, et al. The risk of myocardial ischemia in patients receiving desflurane persus sufentanil anaesthesia for coronary artery bypass graft surgery. Anesthesiology 1992; 77: 47-62.

2 Ebert TJ, Muzi M. Sympathetic hyperactivity during desflurane anesthesia in healthy volunteers. Anesthesiology 1993; 79: 444-53.

3 Weiskopf RB, Eger EI II, Noorani $M$, Daniel $M$. Fentanyl, esmolol, and clonidine blunt the transient cardiovascular stimulation induced by desflurane in 
humans. Anesthesiology 1994; 81: 1350-5.

4 Thomson IR, Bowering JB, Hudson RJ, Frais MA, Rosenbloom $M$. A comparison of desflurane and isoflurane in patients undergoing coronary artery surgery. Anesthesiology 1991; 75: 776-81.

5 Parsons RS, Jones RM, Wrigley $S R$, MacLeod KGA, Platt $M W$. Comparison of desflurane and fentanylbased anaesthetic techniques for coronary artery bypass surgery. Br J Anaesth 1994; 72: 430-8.

6 Leung JM, Levine EH. Left ventricular end-systolic cavity obliteration as a estimate of intraoperative hypovolemia. Anesthesiology 1994; 81: 1102-9.

7 Bergquist BD, Leung JM, Bellows WH. Transesophageal echocardiography in myocardial revascularization: I. Accuracy of intraoperative real-time interpretation. Anesth Analg 1996; 82: 1132-8.

8 American Society of Echocardiography Committee on standards, subcommittee on quantitation of twodimensional echocardiograms. Recommendations for quantitation of the left ventricle by two-dimensional echocardiography. J Am Soc Echocardiogr 1989; 2: 358-67.

9 Leung JM, O'Kelly B, Browner WS, et al. Prognostic importance of postbypass regional wall-motion abnormalities in patients undergoing coronary artery bypass graft surgery. Anesthesiology 1989; 71: 16-25.

10 Yasuda $N$, Weiskopf RB, Cabalan $M K$, et al. Does desflurane modify circulatory responses to stimulation in humans? Anesth Analg 1991; 73: 175-9.

11 Weiskopf RB, Eger EI II, Daniel $M$, Noorani $M$. Cardiovascular stimulation induced by rapid increases in desflurane concentration in humans. Results from activation of tracheopulmonary and systemic receptors. Anesthesiology 1995; 83: 1173-8.

12 Muzi M, Ebert TJ, Hope WG, Robinson BJ, Bell LB. Site(s) mediating sympathetic activation with desflurane. Anesthesiology 1996; 85: 737-47.

13 Bunting $H E$, Kelly $M C$, Milligan $K R$. Effect of nebulized lignocaine on airway irritation and haemodynamic changes during induction of anaesthesia with desflurane. $\mathrm{Br} \mathrm{J}$ Anaesth 1995; 75: 631-3.

14 Gormley WP, Murray JM, Trinick TR. Intravenous lidocaine does not attenuate the cardiovascular and catecholamine response to a rapid increase in desflurane concentration. Anesth Analg 1996; 82: 358-61.

15 Weiskopf RB, Moore MA, Eger EI II, et al. Rapid increase in desflurane concentration is associated with greater transient cardiovascular stimulation than with rapid increase in isoflurane concentration in humans. Anesthesiology 1994; 80: 1035-45.

16 Daniel $M$, Eger EI II, Weiskopf $R B$, Noorani $M$. Propofol fails to attenuate the cardiovascular response to rapid increases in desflurane concentration. Anesthesiology 1996; 84: 75-80.
17 Grant J, Scrutton $M$. Novel -adrenoreceptors primarily responsible for inducing human platelet aggregation. Nature 1979; 277: 659-61.

18 Parker SD, Breslow MJ, Frank SM, et al. Catecholamine and cortisol responses to lower extremity revascularization: correlation with outcome variables. Crit Care Med 1995; 23: 1954-61. 